\title{
A Graphical Game for Cooperative Neighbourhoods of Selfishly Oriented Entities
}

\author{
Antoniou Josephina, Lesta Papadopoulou Vicky, \\ Libman Lavy and Pitsillides Andreas
}

Additional information is available at the end of the chapter

http://dx.doi.org/10.5772/54394

\section{Introduction}

The chapter presents a graphical game of selfishly oriented players, inspired by considering a dense urban residential area where each home unit has its own IEEE 802.11 based wireless access point (AP), deployed without any coordination with other such units. The motivation for this game is to provide a framework where coordination between the game players is desired even though in the real world environment the APs lack any management regarding the efficient utilization of the communication channels and furthermore, it is quite common for a terminal served by one of the APs to be within the signal range of multiple alternative APs. This may not be desirable since APs can be in competition for the same communication resource (radio channel), and since the current standards dictate that at any given time every terminal must be rigidly associated with one particular AP, this situation results in increased interference and consequently a low utilization efficiency of the radio resource, when same or overlapping channels are selected by neighbouring APs.

The graphical game aims to motivates cooperation of the players, i.e. the APs, to overcome the resulting interference because of the unmanaged dense deployment of the APs. In fact, it would be much better for individual APs that are in physical proximity to each other to form groups, where one member of the group would serve the terminals of all group members in addition to its own terminals, so that the other access points of the group can be silent or even turned off, thereby reducing interference and increasing overall Quality of Experience (QoE). In this chapter, these groups include only members whose signal strength is sufficient to serve all group members, so that the access point that would be responsible for serving the terminals of a particular group or neighbourhood could change on a rotating basis, to allow all group members to equally serve and be served. Since there is no centralized entity that can control the APs and force them to form cooperative groups, the creation of such groups must be able to arise from a distributed process where each AP makes its own decisions independently and rationally for the benefit of itself and its terminals. Graphical game theory [2] is an appropriate tool to model such decentralized, topology-dependent schemes. 
In the case of cooperation, i.e. when an AP serves the client of one or more neighbouring APs, in addition to its own clients, it is assumed that there is always enough resource for all clients served, otherwise the particular AP would not be considered as a neighbour. It is reasonable to assume that all APs have an active own set of clients that they serve when active whether they are in cooperation with a neighbour or not. The basic motivation behind the proposed approach, is the inefficiency of wireless communication caused by interference when multiple closely located APs, using the same or overlapping channels operate at the same time. If the clients of all APs are served by only one AP (even if these alternate from time to time), the interference between them is avoided and they receive service much closer to the theoretical limit of the radio resource. Hence, the proposed model esentially aims to capture how the APs may enter cooperation agreements in order to share the serving of each other's clients, in order to eliminate the interference factor caused by each other's transmission from their clients' experience.

In this chapter, we model the idea of cooperative neighbourhhoods as a graphical game and show that there exists motivation towards the cooperation of individual APs, each represented in the model by a dual nature node, a node encapturing both a server (AP) and a client. The strategical decision of such a unit to voluntarily participate in a group where members serve clients of neighbouring nodes in addition to their own, has the property that a unit is more likely to gain more in terms of QoE, than a unit defecting from such cooperation. We will henceforth refer to the proposed graphical game model as the cooperative-neighbourhood game.

\section{Related work}

\subsection{Wireless deployments in urban environments}

The density of wireless networks in urban residential areas is on the rise with more and more home networks being deployed in quite close proximity, enabled by the low cost and easy deployment of off-the-shelf IEEE 802.11 hardware and other personal wireless technologies. It is not uncommon for a wireless station to be within range of dozens of APs [3], competing for the limited number of channels offered by the IEEE 802.11 wireless standard. In this sense, urban areas are becoming similar to campus-like environments; however, in organizations and campuses experts can carefully control and manage interference of access points by planning the setup of the network in advance [4]. On the other hand, wireless networks in urban residential environments have a number of characteristics that make their deployment more challenging. For instance, the network is unplanned, thus aspects of planning such as coverage and interference cannot be controlled. Furthermore, deployments are mostly spontaneous, resulting in uneven density of deployment, the network lacks aspects such as efficient placement of access points, troubleshooting and adapting to network changes such as traffic load, as well as security issues. The authors of [3] use the term chaotic deployments or chaotic networks to refer to such a collection of wireless networks which are unplanned and unmanaged. However, they do mention advantages of such chaotic networks, for instance easily enabling new techniques to determine location [5] or providing near ubiquitous wireless connectivity [6]. The main disadvantage of these chaotic deployments is that interference can significantly affect end-user performance, while being hard to detect [7]. In this chapter we consider a solution based on "virtualization" among the interfering APs, where APs serve each others' clients. The security implications of allowing association of clients across APs from multiple owners are being addressed, e.g. in [8]. In this chapter we 
focus on the incentive aspect of a particular model of cooperation through the use of graphical game theoretic tools, and propose a framework to ensure that the APs are indeed motivated to provide service to each others' clients. In this chapter we focus on the theoretical framework and ignore protocol-specific implementation details.

\subsection{Strategical decision-making in network environmnents}

In this chapter, we consider the interactions between the individual units in dense urban deployments of wireless networks, represented by dual nature vertices in a graph. Describing and analysing interactions between independent, selfish entities is a situation that makes a good candidate to be modeled using the theoretical framework of Game Theory. Game Theory provides appropriate models and tools to handle multiple, interacting entities attempting to make a decision, and seeking a solution state that maximizes each entity's utility, i.e. each entity's quantified satisfaction. Game Theory has been extensively used in networking research as a theoretical decision-making framework, e.g. for cooperative resolution of interactive networking scenarios [1], for routing [9, 10], congestion control [11, 12], resource sharing $[13,14]$, and heterogeneous networks $[15,16]$.

In [17] the authors address the issue of cooperative neighbourhoods by concentrating on the Prisoner's Dilemma/Iterated Prisoner's Dilemma game model and proposing a group strategy to motivate adjacent neighbours into cooperation. The Prisoner's Dilemma and Iterated Prisoner's Dilemma [18] have been a rich source of research since the 1950s. In particular, the publication of Axelrod's book in 1984 [19] was the main driver that boosted the concept to the attention of other areas outside of game theory, as a model for promoting cooperation. The empirical results of the Iterated Prisoner's Dilemma (IPD) tournaments organized by Axelrod have influenced the game theory, machine learning and evolutionary computation communities, showing how features such as adaptivity and group play can result in gains at indiviual level. In fact, adaptive players, learning from the games in which they are involved, are more likely to survive than non-adaptive players in evolutionary IPD [20] and group strategies performed extremely well and defeated well-known strategies in round-robin competitions in the 2004 and 2005 IPD tournaments [21]. In this chapter, the idea of cooperative neighbourhoods is revisited by using the idea of a graph to set the neighbourhood map and defining a game on that graph where cooperation can result in gains for the individual nodes, shown through the usage of the graphical game model in selected examples.

\subsection{Graphical games}

Graphical games are one-shot games that model multi-player interaction in situations where restrictions and influences among the player population may exist. In fact, graphical games are a more efficient representation of one-shot multi-player games that cannot efficiently be represented with the normal form representation of a game (where rows and columns in a table represent the available actions and resulting payoffs of the game players). In addition to providing a better representation for multi-player games, graphical games are meant to capture locality and how the game is affected by the player's positioning.

Graphical games adopt a simple graph-theoretic model, where a game is represented by a graph $G$ in which players are identified with vertices. A player of vertex $i$ has payoffs that are entirely specified by the actions of $i$ and those of its neighbour set in $G$, i.e. the set of 
vertices that have a direct link to $i$. Thus the graph of a game defines structural constraints over players' strategic influences towards other players. To fully describe the graphical game, in addition to the graph itself, the numerical payoff functions to each player must be specified.

There are quite a few gains in the use of graphical games. As mentioned before, in large population games, the graph form simplifies the representation of the game through a rich language in which to state and explore the computational benefits of various restrictions, e.g. topological restrictions, to the game payoffs. Furthermore, the simplified modelling of complex interactive situations as games through the use of the graph-theoretic language, is a tool of studying problems in various disciplines and understanding them through a simple but powerful theoretical framework. As such, we use graphical games in this chapter for modelling and analyzing a multi-entity problem characterized by complex interactions, from the communication networking field. [2]

\section{Introducing the scenario}

Currently, dense residential deployments of home wireless networks consist of uncoordinated APs that serve their terminals individually. The APs do not form groups and share the communication channel, which is an unmanaged common resource, resulting in a low utilization efficiency due to the competition between the APs and the interference it causes. This interference can be reduced if the APs can form groups according to their location, such that any APs belonging to the same group can serve any terminal associated with any of the other group members.

We consider that it is possible for an AP to recognize its neighbourhood from the signals it receives, having a knowledge of the required signal strength thresholds that would serve its terminals in a satisfying manner, i.e. with the required perceived QoE. In such a neighbourhood only one of the APs needs to assume the role of a leader, while the others can remain silent, and thereby minimize the interference and improve the overall QoE for all terminals involved. The role of the leader can be assumed on a rotating basis. Of course, in order to take part in a cooperative neighbourhood, the APs need to be motivated to act cooperatively, i.e. have an incentive to be silent or turned off while it is the turn of another AP to serve, and to serve everyone's terminals once their own turn comes. We show how such a distributed logic can be motivated and sustained in the neighbourhood using a graphical game model where each AP can make an independent decision whether or not to cooperate in such manner with its neighbours.

The interactions in a cooperative neighbourhood can be modelled as a game between the participating units, represented as vertices on a graph that captures through its links the signal received at each node from the neighbouring nodes. Given that each member of the neighbourhood has two choices at any given time: (a) to cooperate with one or more neighbours or (b) serve only its own clients, the graphical game model captures the utility of each node according to the decisions made by the whole neighbourhood, including itself, and provides a framework for scheduling the activation and deactivation times for the servers (APs) of cooperating nodes ${ }^{1}$. Which of the two behaviours to select in each round depends on

\footnotetext{
${ }^{1}$ Note that units may be a part of more than one neighbourhood, i.e. receive a good signal from peers that are in different neighbourhoods, making the scheduling task more challenging. This is part of future work.
} 
the strategy of behaviour that a player has decided to follow during the game. The strategy of each player, i.e. of each unit, is selected such that it results in the highest possible payoff for the particular player, regardless of the strategies selected by the other neighbouring nodes. We refer to such interaction as a cooperative-neighbourhood game.

The chapter uses graphical game theory tools to define a network of APs represented using graph theory, and the relatiosnhip between any two neighbouring APs is represented in terms of the QoE that an AP's client, based on the signal strength received from its neighbouring $\mathrm{AP}$, perceives, if served by the neighbouring AP. In other words, the signal strength that the client of an AP perceives from its neighbour when all other signals are off (which may not always be the case), is captured by the proposed model as the weight on the link joining the two corresponding vertices. Thus, the model represents each AP as a node on a graph and includes weights on the incoming links of each node, based on the signal(s) received at the node from the particular neighbour. We assume that weight is a measurable quantity, which may be obtained with a successive interchange between a node and its neighbours. However, the practical details of such an interchange are protocol design specific, and are out of the scope of this chapter. In the rest of the chapter the term weight represents the received signal strength at a node from a neighbouring node, when all other neighbouring node signals are off.

\section{The graphical game model of a cooperative neighbourhood}

\subsection{The graph}

We consider a set of nodes $V=\left\{v_{1}, v_{2}, v_{3}, \ldots v_{n}\right\}$ located on a plane. Each node is considered as an entity comprising of one server and a constant number of clients. Without loss of generality we assume that each server serves one client, and consequently each node employs dual functionality, i.e. both the functionality of a server and the functionality of a client. Therefore, a server node serves its client node, using broadcast transmission. At a time $t$, we say that node $v_{i}$ is active or $O N$, if its server is broadcasting (to its own client). Otherwise, we say that the node is inactive or OFF.

Consider two nodes, $v_{i}, v_{j}, i \neq j$, which are close enough to detect each other's signals. In particular, node $v_{i}$ may receive information from node $v_{j}$ when it is OFF while node $v_{j}$ is $O N$, i.e. when node $v_{j}$ broadcasts. If the quality of the signal received by node $v_{i}$ from node $v_{j}$ is above some lower bound value assumed by the node $v_{i}$, we may consider that there exists a directed edge from node $v_{j}$ to node $v_{i}$, denoted as $\left(v_{j}, v_{i}\right)$. Moreover, we can quantify the quality of the received signal by having a weight $w\left(v_{j}, v_{i}\right)$ associated with the directed edge $\left(v_{j}, v_{i}\right)$. Normalizing this value we consider that $w\left(v_{j}, v_{i}\right) \in(0,1)$. Finally, this value is analogous to the quality of the received signal, i.e. better received quality corresponds to value of $w\left(v_{j}, v_{i}\right)$ close to 1 . Summing up, this reasoning motivates the following mathematical definition for the two nodes:

Definition 1. Consider two nodes $v_{i}$ and $v_{j}$ such that the two nodes can detect each other's signals and the node $v_{i}$ can receive information from node $v_{j}$ when it is OFF while node $v_{j}$ is $\mathrm{ON}$, of quality above some lower bound value. Then, we assume that there exist a directed edge from node $v_{j}$ to node $v_{i}$, denoted as $\left(v_{j}, v_{i}\right)$ of a positive weight $w\left(v_{j}, v_{i}\right) \in(0,1)$. The weight is analogous to the quality of the received signal at node $v_{i}$. 
Thus, more formally, using the set of nodes $V$, we define a weighted graph $\mathcal{G}=(V, \vec{E}, \vec{W})$, where $\vec{W}:(V \times V) \rightarrow[0,1]$ and $\vec{E} \ni\left(v_{i}, v_{j}\right), i \neq j$, if and only if node $v_{j}$ can receive the signal of node $v_{i}$ when $v_{i}$ is $O N$ and $v_{j}$ is OFF. The measurement of the quality of the received signal by node $v_{i}$ 's client once it is OFF and cooperates with node $v_{j}$, which is $O N$, using edge $\left(v_{i}, v_{j}\right)$, is given by weight $w\left(v_{i}, v_{j}\right)$. Thus, $w\left(v_{i}, v_{j}\right)$ is positive if and only if $\left(v_{i}, v_{j}\right) \in \vec{E}$. We assume that if $w\left(v_{i}, v_{j}\right)>0$, then $w\left(v_{j}, v_{i}\right)>0$ for all nodes $v_{i}, v_{j} \in V, i \neq j$.

\subsection{The time}

We consider a basic unit of time period $T$, e.g. 1 hour, and we split the time period $T$ into $x$ smaller time slots $T_{1}, T_{2}, \ldots T_{x}$ such that for each $T_{k} \in T$ there exists at least one node that in a group of colocated nodes that may alternate between the $O N$ and OFF node, remains $O N$ for the whole time slot $T_{k}$. So, $\bigcup_{T_{k}}=T$ and $T_{k} \cap T_{l}=\varnothing, k \neq l$, i.e. the sum of the time slots is the time period $T$ and no two time slots overlap. By $\left|T_{k}\right|$ we denote the time elapsed from the beginning of time slot $T_{k}$ until the end of the time slot.

Fix a time slot $T_{k}$. Then, for any node $v_{i} \in V$,

$$
\operatorname{Mode}\left(T_{k}, v_{i}\right)= \begin{cases}0, & \text { if node } v_{i} \text { is OFF in time slot } T_{k} \\ 1, & \text { if node } v_{i} \text { is ON in time slot } T_{k} .\end{cases}
$$

So, for node $v_{i}$ the time period $T$ can be partitioned into two sets $O N_{T}\left(v_{i}\right)$ and $\operatorname{OFF}_{T}\left(v_{i}\right)$, where $O N_{T}\left(v_{i}\right)=\left\{T_{k} \mid \operatorname{Mode}\left(T_{k}, v_{i}\right)=O N\right\}$ and $O F F_{T}\left(v_{i}\right)=\left\{T_{k} \mid \operatorname{Mode}\left(T_{k}, v_{i}\right)=O F F\right\}$.

\subsection{Cooperative and non-cooperative neighbours}

Within a given time period $T$, node $v_{i}$ may be in agreement or in cooperation with some of its neighbouring nodes. For any node $v_{j}$, being in agreement with node $v_{i}, i \neq j$ means that node $v_{j}$ broadcasts only when $v_{i}$ does not broadcast and serves the client of node $v_{i}$ in addition to its own client, during the time that node $v_{i}$ is OFF. The set of the neighbours of node $v_{i}$ that are in agreement during time $T$, is denoted by $\operatorname{Coop}_{T}\left(v_{i}\right)$, while the complete set of neighbours of node $v_{i}$ is denoted by $\operatorname{Nei}_{T}\left(v_{i}\right)$, where $\operatorname{Coop}_{T}\left(v_{i}\right) \subseteq \operatorname{Nei}_{T}\left(v_{i}\right)$ and $\operatorname{Mode}\left(T_{k}, v_{i}\right)=1-$ $\operatorname{Mode}\left(T_{k}, v_{j}\right)$. So, $\operatorname{ON}_{T}\left(v_{i}\right)=\operatorname{OFF}_{T}\left(v_{j}\right)$ and $\operatorname{ON}_{T}\left(v_{j}\right)=\operatorname{OFF}_{T}\left(v_{i}\right)$ for each $v_{j} \in \operatorname{Coop}_{T}\left(v_{i}\right)$. On the other hand, the set of neighbours with which $v_{i}$ is not in agreement with is denoted as $\operatorname{NCoop}_{T}\left(v_{i}\right)$, where $\operatorname{NCoop}_{T}\left(v_{i}\right) \subseteq \operatorname{Nei}_{T}\left(v_{i}\right)$ and it may be that $\operatorname{Mode}\left(T_{k}, v_{i}\right)=\operatorname{Mode}\left(T_{k}, v_{j}\right)$ where $v_{j} \in \operatorname{NCoop}_{T}\left(v_{i}\right)$.

\subsection{Interference and quality of experience}

\subsubsection{Received Signal}

We are interested in measuring the QoE received by the client of node $v_{i}$ through the signal strength received at node $v_{i}$, at any time slot $T_{k}$ during the time period $T$. The received QoE, considering both cooperative and non-cooperative neighbours during time period $T$, is approximated, for simplicity, as the summation of the edge weights of all interfering nodes. We denote this quantity as $\operatorname{rec}_{T_{k}}\left(v_{i}\right)$. We also distinguish two kinds of signals received at node $v_{i}$ : 
- the signal received when node $v_{i}$ is $O N$ denoted as $O N r e c S_{T_{k}}\left(v_{i}\right)$, and

- the signal received when node $v_{i}$ is OFF denoted as OFFrec $S_{T_{k}}\left(v_{i}\right)$.

At any time $T_{k}$ the node is either $O N$ or OFF. Thus,

$$
\operatorname{rec}_{T_{k}}\left(v_{i}\right)= \begin{cases}\operatorname{OFFrec} S_{T_{k}}\left(v_{i}\right), & \text { if node } v_{i} \text { is OFF } \\ \operatorname{ONrec} S_{T_{k}}\left(v_{i}\right), & \text { if node } v_{i} \text { is ON }\end{cases}
$$

\subsubsection{ON operation}

Fix a time slot $T_{k} \in T$. When node $v_{i}$ is $O N$ and none of its neighbours broadcast at the same time, the client of node $v_{i}$ receives the broadcast signal in the best quality (no interference) and hence experiences the best possible QoE. We assume that top QoE is measured by a unit. Therefore, we set the experienced QoE by the node's client functionality to be equal to 1 in this case.

When node $v_{i}$ is $O N$, none of its neighbours in cooperation, i.e. set $\operatorname{Coop}_{T}\left(v_{i}\right)$ are $O N$ at the same time. However, some of its neighbours not in cooperation may be $O N$, i.e. set $\operatorname{NCoop}_{T}\left(v_{i}\right)$. In the second case, interference occurs and the signal received by node $v_{i}$ is degraded causing a degraded QoE. We consider the degradation to be analogous to the strength of the signal of neighbour $v_{j}$ received at node $v_{i}$ and is captured by the weight $w\left(v_{j}, v_{i}\right)$ of edge $\left(v_{j}, v_{i}\right)$. This degradation is also increased as more than one non-cooperative neighbours broadcast at the same time as node $v_{i}$. For simplicity, we consider the degradation to be analogous to the strengths of their signals received at node $v_{i}$.

Thus,

$$
\operatorname{ONrec}_{T_{k}}\left(v_{i}\right)=\max \left\{0,1-\sum_{v_{j} \in N e i_{T_{k}}\left(v_{i}\right)} w\left(v_{j}, v_{i}\right) \cdot \operatorname{Mode}\left(T_{k}, v_{j}\right)\right\}
$$

\subsubsection{OFF operation}

On the other hand, when node $v_{i}$ is $O F F$, the quality of the signal received at node $v_{i}$, and hence the QoE experienced, depends on the number of neighbours in cooperation with node $v_{i}$, that are $O N$ at time $T_{k}$ and serving the clients of the nodes in cooperation that are OFF, including the client of node $v_{i}$. If there exists one such neighbouring node $v_{j}$ that is $O N$, the quality of the signal is captured by the weight $w\left(v_{j}, v_{i}\right)$ of edge $\left(v_{j}, v_{i}\right)$. However, if there exist more than one neighbouring nodes not in cooperation with node $v_{i}$ that are $O N$ at the same time, this results in interference received at node $v_{i}$, which degrades the experienced QoE at the client of node $v_{i}$. This chapter considers the QoE degradation to be analogous to the strengths of the received signals.

We assume that node $v_{i}$ is tuned to the strongest signal of its neighbours in set $\operatorname{Coop}_{T}\left(v_{i}\right)$. Note that the quality of this signal is also degraded by the sum of the received signals from cooperative and non-cooperative neighbours that are $O N$ at the same time as node $v_{i}$ is. The set of neighbouring nodes that are $O N$ at the same time as node $v_{i}$ are considered to be non-cooperative. 
Thus,

$$
\begin{aligned}
\operatorname{OFFrec}_{T_{k}}\left(v_{i}\right) & =\max \left\{0,\left(\max _{v_{j} \in \operatorname{Coop}_{T_{k}}\left(v_{i}\right)}\left\{w\left(v_{j}, v_{i}\right) \cdot \operatorname{Mode}\left(T_{k}, v_{j}\right)\right\}\right.\right. \\
& \left.\left.-\sum_{v_{h} \in \operatorname{Nei}_{T_{k}}\left(v_{i}\right), v_{h} \neq m} w\left(v_{h}, v_{i}\right) \cdot \operatorname{Mode}\left(T_{k}, v_{h}\right)\right)\right\},
\end{aligned}
$$

where $m \in V$ and $w(m, i)=\max _{j \in \operatorname{Coop}_{\sigma}(i)}\left\{w(j, i) \cdot \operatorname{Mode}\left(T_{k}, j\right)\right\}$. So, summing up for the time period $T$ :

$$
\operatorname{recS}_{T}\left(v_{i}\right)=\sum_{\operatorname{Mode}\left(T_{k}, v_{i}\right)=\text { ON }} \operatorname{ONrec}_{T_{k}}\left(v_{i}\right) \cdot\left|T_{k}\right|+\sum_{\operatorname{Mode}\left(T_{k}, v_{i}\right)=\text { OFF }} \operatorname{OFFrec}_{T_{k}}\left(v_{i}\right) \cdot\left|T_{k}\right|
$$

\subsection{The cooperative neighbourhood game}

We consider an one-shot strategic game resulting from the described scenario in which the players of the game are the nodes (servers). Given the decisions of the nodes whether to operate in $O N$ or OFF operation during each time slot $T_{k} \in T$, the utility of player $v_{i}$ is the received signal of $v_{i}$ 's client during time period $T$, given by $\operatorname{rec} S_{T}\left(v_{i}\right)$.

Thus, more formally we define:

Definition 2. The game $G(V, E, W)$ is defined as follows. The set of players of the game is the set $V$. For simplicity, we define $V=\{1, \ldots, n\}$. A profile $\sigma$ of the game is associated with the basic time period $T$ of the scenario described. $T$ is split into time slots $T_{1}, T_{2}, \ldots T_{x}$, such that $\bigcup_{T_{k}}=T$ and $T_{k} \cap T_{l}=\varnothing, k \neq l, T_{k}$ corresponding to the smallest time slot where we may have alterations between $O N$ and OFF operations of the nodes.

The strategies of the players in a profile $\sigma$ are defined as follows:

The strategy of player (node) $i$ is given by $\sigma_{i}=\left(\operatorname{Mode}_{\sigma}(T), \operatorname{Coop}_{\sigma}(i)\right)$, where $\operatorname{Mode}_{\sigma}(T)$, a vector of $0 \mathrm{~s}$ and $1 \mathrm{~s}$, such that $\operatorname{Mode}_{\sigma}\left(T_{k}, i\right)=0$ or 1 , based on whether node $i$ operates in ON or OFF mode during time slot $T_{k}$, for each $T_{k} \in T$. $\operatorname{Coop}_{\sigma}\left(v_{i}\right) \subseteq \operatorname{Nei}_{\sigma}\left(v_{i}\right)$ is the set of neighbouring nodes of node $i$, with which node $i$ has decided to cooperate with in $\sigma$. Cooperation means that for each such cooperative neighbour $j$ of node $i, \operatorname{Mode}_{\sigma}\left(T_{k}, i\right)=$ $1-\operatorname{Mode}_{\sigma}\left(T_{k}, j\right)$ for each $T_{k} \in T$, and the two nodes are in agreement to serve each other's client.

For player (node) $i$ denote,

$$
\operatorname{maxCoop}_{\sigma}\left(T_{k}, i\right)=\left\{m \in V \mid \operatorname{Mode}\left(T_{k}, m\right)=1, w(m, i)=\max _{j \in \operatorname{Coop}_{\sigma}(i)}\left\{w(j, i) \cdot \operatorname{Mode}\left(T_{k}, j\right)\right\}\right.
$$

Then, the utility of player (node) $i$, representing the signal received as expressed in equations (2), (3), (4), is given by:

$$
U_{\sigma}(i)=\sum_{T_{k} \in \mathrm{ONU}_{T}(i)} \mathrm{ONU}_{T_{k}}(i) \cdot\left|T_{k}\right|+\sum_{T_{k} \in \mathrm{OFFU}_{T}(i)} \operatorname{OFFU}_{T_{k}}(i) \cdot\left|T_{k}\right|
$$

where,

$$
\mathrm{ONU}_{T_{k}}(i)=\max \left\{0,1-\sum_{j \in N e i(i)} w(j, i) \cdot \operatorname{Mode}\left(T_{k}, j\right)\right\}
$$

and

$$
\begin{aligned}
& \operatorname{OFFU}_{T_{k}}(i)=\quad \max \left\{0, w\left(\max \operatorname{Coop}_{\sigma}\left(T_{k}, i\right), i\right)\right. \\
& \left.-\sum_{h \in \operatorname{Nei}(i), h \neq \max \operatorname{Coop}_{\sigma}\left(T_{k}, i\right)} w(h, i) \cdot \operatorname{Mode}\left(T_{k}, h\right)\right\}
\end{aligned}
$$




\section{A usage example}

In this section, we consider a simple scenario to demonstrate the interaction between two interacting neighbours. We juxtapose the situation where the two neighbours do not cooperate versus the case where the two neighbours cooperate, and we look for the time split between $O N$ and OFF time slots for which cooperation is beneficial. We consider two case studies for the two nodes, where in the first case the time split between $O N$ and OFF times is such that the $O N$ time for one of the nodes is much larger than its OFF time. In the second case, we select equal $O N$ and $O F F$ times. The motivation behind the usage example is to determine the role of a particular time split towards the decision of the nodes to cooperate. We discover that in both cases there exists a motivation to cooperate, which leads to the generalization of these findings for the cooperation of two nodes in the subsequent section.

\subsubsection{Case 1}

The example first considers the case of the two nodes not in cooperation. Each node is $O N$, with its own client receiving the maximum signal from its own server, i.e. 1, while simultaneously the adjacent node is $O N$ causing a continuous interference.

Consider the case where $w(2,1)=0.7$, and $w(1,2)=0.6$. Hence according to the utility defined in equation (5), $\mathrm{ONU}_{T}(1)=1-w(2,1)=1-0.7=0.3$ and $O F F U_{T}(1)=0$. Also, $\mathrm{ONU}_{T}(2)=1-w(1,2)=1-0.6=0.4$ and $\mathrm{OFFU}_{T}(2)=0$.

In the case that the two nodes are in cooperation, then they alternate between states of $O N$ and OFF times, while each node serves the client of its neighbour during its ON time, while its client is being served by the neighbour's server, during its OFF time. The utilities for nodes 1 and 2 are given in equation (5). Given that we want to consider a time split of non-equal times, we arbitrarily select the first time split of the total time period $T$, to be such that $T=T_{1}+T_{2}$, where $T_{1}=0.2, T_{2}=0.8$. Note that in this setting $\operatorname{Max} \operatorname{Coop}_{\sigma}\left(T_{1}, 2\right)=1$ and $\operatorname{Max} \operatorname{Coop}_{\sigma}\left(T_{2}, 1\right)=2$. Therefore,

$$
\begin{aligned}
U_{\sigma}(1) & =\text { ONU }_{T_{1}}(1)+\text { OFFU }_{T_{2}}(1) \\
& =1 \times 0.8+0.7 \times 0.2=0.94 \\
U_{\sigma}(2) & =\text { ONU }_{T_{1}}(2)+\text { OFFU }_{T_{2}}(2) \\
& =1 \times 0.2+0.6 \times 0.8=0.68
\end{aligned}
$$

Therefore, we observe that even for the given weights, the cooperation option results in improved utility for both nodes 1, 2, compared to the non-cooperative values, therefore, the use of cooperation results in important gains.

\subsubsection{Case 2}

In this section, we consider a similar case scenario with two interacting nodes 1 and 2 but we modify the proposed time split of the total time period $T$, to be such that $T=T_{1}+T_{2}$ and $T_{1}=T_{2}$, where $T_{1}=0.5, T_{2}=0.5$.

In the case of non-cooperation, as previously, each node is $O N$, with its own client receiving the maximum signal from its own server, i.e. 1, while simultaneously the adjacent node is $O N$ 
causing a continuous interference. Since $w(2,1)=0.7$, and $w(1,2)=0.6$, then similarly to case 1 , according to equation (5), $\operatorname{ONU}_{T}(1)=1-w(2,1)=1-0.7=0.3$ and $\operatorname{OFFU}_{T}(1)=0$. Also, $\mathrm{ONU}_{T}(2)=1-w(1,2)=1-0.6=0.4$ and $O F F U_{T}(2)=0$.

In the case of cooperation, note that as in case $1, \operatorname{Max} \operatorname{Coop} \sigma\left(T_{1}, 2\right)=1$ and $\operatorname{Max} \operatorname{Coop}_{\sigma}\left(T_{2}, 1\right)=$ 2. Hence,

$$
\begin{aligned}
U_{\sigma}(1)= & \text { ONU }_{T_{0.9}}\left(n_{1}\right)+\operatorname{OFFU}_{T_{0.1}}\left(n_{2}\right) \\
= & 1 \times 0.5+0.7 \times 0.5=0.85
\end{aligned}
$$

We observe again that cooperation results in improved utility for both nodes 1,2 , compared to the non-cooperative utility values. Therefore, we have seen that for the both cases, the cooperation is the most profitable option. Next, we discuss and prove that for weights above the value of 0.5 , cooperation is the most profitable option regardless of the particular time split between the $O N$ and OFF times for the situation of two neighbouring nodes.

\section{Nash Equilibrium for two neighbouring nodes}

For ease of exposition, we consider the simplest case where the cooperative-neighbourhood game is played between two adjacent nodes 1 and 2, where the signal broadcast by each node's server is received by the other node's client in addition to the broadcasting node's own client. Let the weights on their incident edges denoted by $w(1,2)$ and $w(2,1)$. Remember that the utility of each node is defined in equations (5), (6) and (7).

Note that if a node decides to cooperate with at least one neighbour, then it is OFF for some of the time. On the other hand, if it does not cooperate with any neighbour at any time, then it is $O N$ for the whole time $T$. In the following we compare values of utility in cooperation and non-cooperation of the two nodes and show necessary conditions in order to get a Nash Equilibrium for the two nodes.

\subsection{Two interacting neighbouring nodes}

Next, we compare the utilities of node 1 and node 2 in both the case where they cooperate and the case they do not cooperate, and we show necessary conditions for a cooperative profile in order to be a Nash Equilibrium.

\subsubsection{Case 1: No cooperation of both nodes}

In case 1 , we consider that both nodes are $O N$ for the whole duration of time period $T$, i.e. they do not cooperate with each other. Let $\sigma$ be the resulting profile. Then, by equation (5),

$$
\begin{aligned}
& U_{\sigma}(1)=\quad O N U_{T}(1) \\
& =(1-w(2,1) \cdot \operatorname{Mode}(T, 2)) \cdot T \\
& =\quad(1-w(2,1)) \cdot T
\end{aligned}
$$


Also,

$$
\begin{array}{rlc}
U_{\sigma}(2) & = & \operatorname{ONU}_{T}(2) \\
& =(1-w(1,2) \cdot \operatorname{Mode}(T, 1)) \cdot T \\
& = & (1-w(1,2)) \cdot T
\end{array}
$$

\subsubsection{Case 2: Cooperation of the two nodes}

We consider here the case where the two nodes agreed to cooperate in T. Assume without loss of generality that the two nodes agreed to split $T$ into two parts, $T_{1}$ and $T_{2}$, such that $T_{1} \cap T_{2}=\varnothing$ and $T=T_{1} \cup T_{2}$ and $\operatorname{Mode}\left(T_{1}, 1\right)=1=1-\operatorname{Mode}\left(T_{1}, 2\right)$ and $\operatorname{Mode}\left(T_{2}, 2\right)=1=$ $1-\operatorname{Mode}\left(T_{2}, 1\right)$. Let $\sigma$ be the resulting profile. Then, by equation (5),

$$
\begin{aligned}
& U_{\sigma}(1)=O N U_{T_{1}}(1) \cdot T_{1}+O F F U_{T_{2}}(2) \cdot T_{2} \\
& =T_{1}+w(2,1) \cdot \operatorname{Mode}\left(T_{2}, 2\right) \cdot T_{2} \\
& =\quad T_{1}+w(2,1) \cdot T_{2}
\end{aligned}
$$

Similarly, we show that,

$$
U_{\sigma}(2)=T_{2}+w(1,2) \cdot T_{1}
$$

\subsection{Equilibrium for the two nodes}

We prove,

Theorem 6.1. Assume two interacting nodes 1,2 with $w(1,2)$ and $w(2,1)$ the weights of the link between them. Then cooperation when the two nodes split $T$ into two sets $T_{1}, T_{2}$ such that $T_{1} \cap T_{2}=\varnothing$ and $T=T_{1} \cup T_{2}$ is a Nash Equilibrium for the nodes if both $w(1,2)$ and $w(2,1) \geq 0.5$.

Proof. We first show that if a profile $\sigma$ satisfies the requirements of the theorem then it is a Nash Equilibrium. Assuming cooperation, the utilities of the nodes are given by equations (14) and (15) above. We now show that any unilateral alternations of any of the two nodes do not increase their utilities. Each of the two nodes has two possible alternations:

1. To increase its $\mathrm{ON}$ period.

2. To decrease its $\mathrm{ON}$ period.

We first consider the second option, i.e. for the node to decrease its ON period. Let $t$ be the increased time in which node 1 is OFF. Let $\sigma^{\prime}$ be the resulting profile.

Let $T_{1}^{\prime}, T_{2}^{\prime}$ be the new split of $T$. Consider first node 1 . Then, $\left|T_{1}\right|^{\prime}=\left|T_{1}\right|-|t|,|t|>0$ and $\left|T_{2}\right|^{\prime}=\left|T_{2}\right|+|t|$. Note that during time period $t$, node 1 will receive:

$$
\begin{array}{rlrl}
U_{\sigma^{\prime}}(1) & = & \text { ONU }_{T_{1}^{\prime}}(1) \cdot T_{1}^{\prime}+\text { OFFU }_{T_{2}^{\prime}}(2) \cdot T_{2}^{\prime} \\
& =\left(\left|T_{1}\right|-|t|\right)+w(2,1) \cdot \operatorname{Mode}\left(T_{2}, 2\right) \cdot T_{2}+w(2,1) \cdot \operatorname{Mode}(t, 2) \cdot t \\
& = & \left(\left|T_{2}\right|-|t|\right)+w(2,1) \times 1 \cdot T_{2}+w(2,1) \cdot 0 \\
& = & \left|T_{1}\right|-|t|+w(2,1) \cdot T_{2} \\
& = & U_{\sigma}(1)-|t|<U_{\sigma}(1), \text { by equation (14) since }|t|>0 .
\end{array}
$$


Thus node 1 has no gain if it changes according to the second option. Similarly, we can show that $U_{\sigma^{\prime}}(2)<U_{\sigma}(2)$. Therefore, the second option of a node decreasing its ON period does not result in some gain for any of the two nodes 1 or 2 .

Next, we consider the first option of a node to increase its ON period. Let $T_{1}^{\prime}, T_{2}^{\prime}$ be the new split of $T, T_{1}^{\prime} \cup T_{2}^{\prime}=T, T_{1}^{\prime} \cap T_{2}^{\prime}=\varnothing$. Assume that there exists some $t>0$, such that without loss of generality, node 1 increases its ON time period to $\left|T_{1}\right|^{\prime}=\left|T_{1}\right|+|t|$ and $\left|T_{2}\right|^{\prime}=\left|T_{2}\right|+|t|$. Note that nothing changes for node 2, i.e. $\operatorname{Mode}\left(T_{1}, 2\right)=O F F$ and $\operatorname{Mode}\left(T_{2}, 2\right)=O N$. Also, $\operatorname{Mode}(t, 2)=O N$.

Then the utility of node 1 in $\sigma^{\prime}$ by equation (5) is,

$$
\begin{aligned}
U_{\sigma^{\prime}}(1) & =\quad \operatorname{ONU}_{T_{1}^{\prime}}(1) \cdot T_{1}^{\prime}+\operatorname{OFFU}_{T_{2}^{\prime}}(2) \cdot T_{2}^{\prime} \\
& =1 \cdot T_{1}+(1-w(2,1)) \cdot t+w(2,1) \cdot\left(\left|T_{2}\right|-|t|\right) \\
& =\quad\left(\left|T_{1}\right|+w(2,1) \cdot T_{2}\right)+|t|-2|t| \cdot w(2,1) \\
& =\quad U_{\sigma}(1)+|t|-2|t| \cdot w(2,1), \quad \text { by equation (15) since }|t|>0 .
\end{aligned}
$$

In order for $\sigma^{\prime}$ to be a better response for mode 1 , it must be that:

$$
\begin{gathered}
U_{\sigma^{\prime}}(1)>U_{\sigma}(1) \\
U_{\sigma^{\prime}}(1)-U_{\sigma}(1)>0, \text { by equation }(17) \\
U_{\sigma}(1)+|t|-2|t| \cdot w(2,1)-U_{\sigma}(1)>0 \\
2|t| \cdot w(2,1)<|t|
\end{gathered}
$$

Therefore,

$$
w(2,1)<\frac{1}{2}
$$

A contradiction, since $w(2,1)>\frac{1}{2}$ by assumption.

Similarly, we can show that for node 2 to benefit from increasing its ON time, it should be that $w(1,2)<\frac{1}{2}$, a contradiction by assumption.

Thus, if any of the nodes 1, 2 unilaterally alters its strategy to the first option of increasing its $\mathrm{ON}$ time, then profile $\sigma^{\prime}$ does not result to a better response than the strategy in $\sigma$. Therefore, $\sigma$ is a Nash Equilibrium.

The theorem is now complete.

\section{An example game profile}

Let Figure 1 illustrate the graph of connected nodes representing a neighbourhood of access points, where access points that are neighbours, i.e. that can serve each other's clients, are joined in the given graph through bidirectional links, the weights for each link given.

Let a time split $T=T_{1} \cup T_{2} \cup T_{3} \cup T_{4}$, where $T_{i} \cap T_{j}=\varnothing$ and $T_{1}=T_{2}=T_{3}=T_{4}$. Assuming $|T|=1,\left|T_{1}\right|=\left|T_{2}\right|=\left|T_{3}\right|=\left|T_{4}\right|=\frac{1}{4}$, let the following mode operations as illustrated in Table 1. 


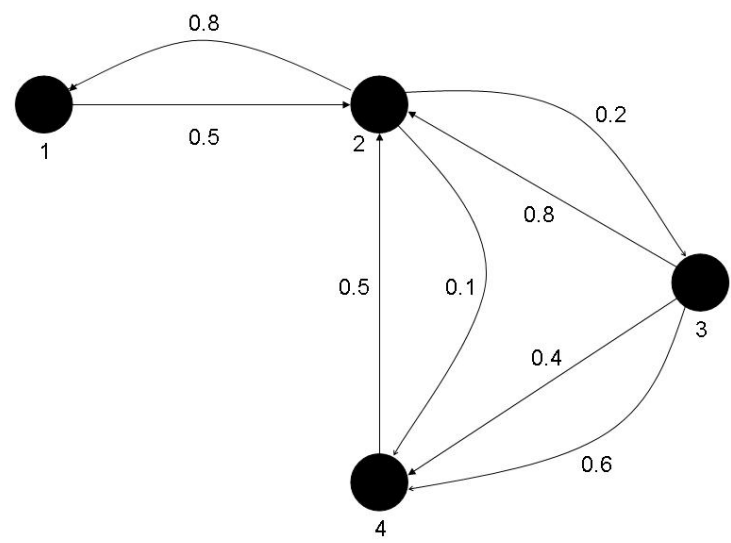

Figure 1. An example graph of the cooperative neighbourhood game

\begin{tabular}{ll}
\hline \hline Node ID & Mode Operation \\
\hline Node 1 & $\operatorname{Mode}\left(T_{1}, 1\right)=\operatorname{Mode}\left(T_{2}, 1\right)=\operatorname{Mode}\left(T_{3}, 1\right)=0$ \\
\hline $\operatorname{Mode}\left(T_{4}, 1\right)=1$ \\
\hline Node 2 & $\operatorname{Mode}\left(T_{1}, 2\right)=1$ \\
\hline $\operatorname{Mode}\left(T_{2}, 2\right)=\operatorname{Mode}\left(T_{3}, 2\right)=\operatorname{Mode}\left(T_{4}, 2\right)=0$ \\
\hline Node 3 & $\operatorname{Mode}\left(T_{1}, 3\right)=\operatorname{Mode}\left(T_{2}, 3\right)=0$ \\
\hline $\operatorname{Mode}\left(T_{3}, 3\right)=\operatorname{Mode}\left(T_{4}, 3\right)=1$ \\
\hline
\end{tabular}

Table 1. Mode operations on Nodes 1-4

Next, Table 2 presents the cooperative and non-cooperative sets of neighbours for each node, based on the example and considering that nodes which have a link between them are potential cooperative neighbours.

\begin{tabular}{lcc}
\hline \hline Node ID & Cooperative Set & Non-Cooperative Set \\
\hline Node 1 & Coop $_{\sigma}(1)=\{2\}$ & NCoop $_{\sigma}(1)=\{\varnothing\}$ \\
\hline Node 2 & $\operatorname{Coop}_{\sigma}(2)=\{1,3,4\}$ & NCoop $_{\sigma}(2)=\{\varnothing\}$ \\
\hline Node 3 & Coop $_{\sigma}(3)=\{2\}$ & NCoop $_{\sigma}(3)=\{4\}$ \\
\hline Node 4 & Coop $_{\sigma}(4)=\{2\}$ & NCoop $_{\sigma}(4)=\{3\}$ \\
\hline \hline
\end{tabular}

Table 2. Cooperative and Non-Cooperative Sets for Nodes 1-4 
We now compute the utilities of nodes 1, 2, 3 and 4, starting from node 1 . Note that for node 1 ,

$$
\begin{aligned}
& \max \operatorname{Coop}_{\sigma}\left(T_{1}, 1\right)= \\
& \max \operatorname{Coop}_{\sigma}\left(T_{2}, 1\right)=\max \operatorname{Coop}_{\sigma}\left(T_{3}, 1\right)=\{\varnothing\}
\end{aligned}
$$

Thus,

$$
\begin{array}{rlrl}
U_{\sigma}(1) & =\text { ONU }_{T_{4}}(1) \cdot\left|T_{4}\right|+\operatorname{OFFU}_{T_{1}}(1) \cdot\left|T_{1}\right|+\operatorname{OFFU}_{T_{2}}(1) \cdot\left|T_{2}\right|+\text { OFFU }_{T_{3}}(1) \cdot\left|T_{3}\right| \\
& = & \max \left\{0,\left(1-\sum_{i \in \varnothing} w(i, 1)\right) \cdot \operatorname{Mode}\left(T_{4}, j\right)\right\} \cdot\left|T_{4}\right| \\
+ & \max \left\{0, w(2,1)-\sum_{h \in\{2\}, h \neq 2} w(h, 1) \cdot \operatorname{Mode}\left(T_{1}, h\right)\right\} \cdot\left|T_{1}\right| \\
+ & \max \left\{0,0-\sum_{h \in\{2\}, h \neq\{\varnothing\}} w(h, 1) \cdot \operatorname{Mode}\left(T_{2}, h\right)\right\} \cdot\left|T_{2}\right| \\
+ & \max \left\{0,0-\sum_{h \in\{2\}, h \neq\{\varnothing\}} w(h, 1) \cdot \operatorname{Mode}\left(T_{3}, h\right)\right\} \cdot\left|T_{3}\right| \\
= & 1 \cdot\left|T_{4}\right|+w(2,1) \cdot\left|T_{1}\right| \\
= & 0.25+0.80 \cdot 0.25=0.45
\end{array}
$$

We proceed to compute the utility of node 2. Note first that,

$$
\begin{aligned}
& \operatorname{maxCoop}_{\sigma}\left(T_{2}, 2\right)=\varnothing \\
& \max \operatorname{Coop}_{\sigma}\left(T_{3}, 2\right)=\{3\} \\
& \operatorname{maxCoop}_{\sigma}\left(T_{4}, 2\right)=\{3\}
\end{aligned}
$$

Thus,

$$
\begin{aligned}
& U_{\sigma}(2)=O N U_{T_{1}}(2) \cdot\left|T_{1}\right|+O F F U_{T_{2}}(2) \cdot\left|T_{2}\right|+O F F U_{T_{3}}(2) \cdot\left|T_{3}\right|+O F F U_{T_{4}}(2) \cdot\left|T_{4}\right| \\
& =\quad \max \left\{0,1-\sum_{j \in\{1,3,4\}} w(j, 2) \cdot \operatorname{Mode}\left(T_{1}, j\right)\right\} \cdot\left|T_{1}\right| \\
& +\quad \max \left\{0,0-\sum_{h \in 1,3,4, h \neq\{\varnothing\}} w(h, 1) \cdot \operatorname{Mode}\left(T_{2}, h\right)\right\} \cdot\left|T_{2}\right| \\
& +\quad \max \left\{0, w(3,2)-\sum_{h \in 1,3,4, h \neq\{3\}} w(h, 2) \cdot \operatorname{Mode}\left(T_{3}, h\right)\right\} \cdot\left|T_{3}\right| \\
& +\quad \max \left\{0, w(3,2)-\sum_{h \in 1,3,4, h \neq\{3\}} w(h, 2) \cdot \operatorname{Mode}\left(T_{4}, h\right)\right\} \cdot\left|T_{4}\right| \\
& =\left|T_{1}\right|+\max \{0, w(3,2)-(w(4,2) \cdot 1+w(1,2) \cdot 0)\} \cdot\left|T_{3}\right| \\
& +\quad \max \{0, w(3,2)-(w(4,2) \cdot 1+w(1,2) \cdot 1)\} \cdot\left|T_{4}\right| \\
& =0.25+\max \{0,(0.8-0.5)\} \cdot 0.25+0 \\
& =\quad 0.25+0.30 \cdot 0.25=\frac{13}{40}
\end{aligned}
$$


Next, we proceed to node 3 . Note that,

$$
\begin{aligned}
& \max \operatorname{Coop}_{\sigma}\left(T_{1}, 3\right)=\{2\} \\
& \max \operatorname{Coop}_{\sigma}\left(T_{2}, 3\right)=\varnothing
\end{aligned}
$$

Thus,

$$
\begin{aligned}
& U_{\sigma}(3)=O N U_{T_{3}}(3) \cdot\left|T_{3}\right|+O_{N} U_{T_{4}}(3) \cdot\left|T_{4}\right|+O F F U_{T_{1}}(3) \cdot\left|T_{1}\right|+O F F U_{T_{2}}(3) \cdot\left|T_{2}\right| \\
& =\quad \max \left\{0,1-\sum_{j \in\{2,4\}} w(j, 3) \cdot \operatorname{Mode}\left(T_{3}, j\right)\right\} \cdot\left|T_{3}\right| \\
& +\quad \max \left\{0,1-\sum_{j \in\{2,4\}} w(j, 3) \cdot \operatorname{Mode}\left(T_{4}, j\right)\right\} \cdot\left|T_{4}\right| \\
& +\quad \max \left\{0, w(2,3)-\sum_{h \in 2,4, h \neq 2} w(h, 3) \cdot \operatorname{Mode}\left(T_{1}, h\right)\right\} \cdot\left|T_{1}\right| \\
& +\quad \max \left\{0,0-\sum_{h \in 2,4, h \neq\{\varnothing\}} w(h, 3) \cdot \operatorname{Mode}\left(T_{2}, h\right)\right\} \cdot\left|T_{2}\right| \\
& =\max \{0,(1-w(4,3) \cdot 1)\} \cdot\left|T_{3}\right|+\max \{0,(1-w(4,3) \cdot 1)\} \cdot\left|T_{4}\right| \\
& +\quad \max \{0,(w(2,3)-w(4,3) \cdot 0)\} \cdot\left|T_{1}\right| \\
& +\quad \max \left\{0,\left(0-\sum_{h \in\{2,4\}, h \neq\{\varnothing\}} \cdot 0\right)\right\} \cdot\left|T_{2}\right| \\
& =\quad w(1-w(4,3)) \cdot\left|T_{3}\right|+(1-w(4,3)) \cdot\left|T_{4}\right|+w(2,3) \cdot\left|T_{1}\right|+0 \\
& =\quad(1-0.6)(0.25)+(1-0.6)(0.25)+(0.2)(0.25)=1 \cdot \frac{1}{4}
\end{aligned}
$$

Finally, we compute the utility of node 4 . Note that,

$$
\begin{aligned}
& \max \operatorname{Coop}_{\sigma}\left(T_{1}, 4\right)=\{2\} \\
& \max \operatorname{Coop}_{\sigma}\left(T_{2}, 4\right)=\varnothing
\end{aligned}
$$

Thus,

$$
\begin{aligned}
& U_{\sigma}(4)=\mathrm{ONU}_{T_{3}}(4) \cdot\left|T_{3}\right|+\mathrm{ONU}_{T_{4}}(4) \cdot\left|T_{4}\right|+\mathrm{OFFU}_{T_{1}}(4) \cdot\left|T_{1}\right|+O F F U_{T_{2}}(4) \cdot\left|T_{2}\right| \\
& =\quad \max \left\{0,1-\sum_{j \in\{2,3\}} w(j, 4) \cdot \operatorname{Mode}\left(T_{3}, j\right)\right\} \cdot\left|T_{3}\right| \\
& +\quad \max \left\{0,1-\sum_{j \in\{2,3\}} w(j, 4) \cdot \operatorname{Mode}\left(T_{4}, j\right)\right\} \cdot\left|T_{4}\right| \\
& +\quad \max \left\{0, w(2,3)-\sum_{h \in\{2,3\}, h \neq 2} w(h, 4) \cdot \operatorname{Mode}\left(T_{1}, h\right)\right\} \cdot\left|T_{1}\right| \\
& \left.+\quad \max \left\{0,0-\sum_{h \in\{2,3\}, h \neq\{\varnothing\}} w(h, 4) \cdot 0\right)\right\} \cdot\left|T_{2}\right| \\
& =\quad w(1-w(3,4)) \cdot\left|T_{3}\right|+(1-w(3,4)) \cdot\left|T_{4}\right|+w(2,4) \cdot\left|T_{1}\right|+0 \\
& =\quad(1-0.4)(0.25)+(1-0.4)(0.25)+(0.1)(0.25)=1.3 \cdot \frac{1}{4}
\end{aligned}
$$


Now, let's investigate whether profile $\sigma$ is a Nash Equilibrium. In order for $\sigma$ to be a Nash Equilibrium for player $i, i$ 's action in $\sigma$ must be a best response action (to the current actions of the best of the players).

Consider player 2. Assume that it increases its $O N$ operation and that it remains $O N$ during time slot $T_{4}$. Then let $\sigma^{\prime}$ be the modified profile. Note that the rest of the players act as in $\sigma$.

$$
\begin{array}{rlr}
U_{\sigma^{\prime}}(2) & = & \operatorname{ONU}_{T_{1}}(2) \cdot\left|T_{1}\right|+\operatorname{OFFU}_{T_{2}}(2) \cdot\left|T_{2}\right| \\
+ & \quad \operatorname{OFFU}_{T_{3}}(2) \cdot\left|T_{3}\right|+\operatorname{ONU}_{T_{4}}(2) \cdot\left|T_{4}\right| \\
& = & \max \left\{0,1-\sum_{j \in\{1,3,4\}} w(j, 2) \cdot \operatorname{Mode}\left(T_{1}, j\right)\right\} \cdot\left|T_{1}\right| \\
+ & \max \left\{0,0-\sum_{h \in 1,3,4, h \notin \varnothing} w(h, 1) \cdot \operatorname{Mode}\left(T_{2}, h\right)\right\} \cdot\left|T_{2}\right| \\
+ & \max \left\{0, w(3,2)-\sum_{h \in 1,3,4, h \neq 3} w(h, 2) \cdot \operatorname{Mode}\left(T_{3}, h\right)\right\} \cdot\left|T_{3}\right| \\
+ & \max \left\{0,1-\sum_{j \in 1,3,4} w(j, 2) \cdot \operatorname{Mode}\left(T_{2}, j\right)\right\} \cdot\left|T_{4}\right| \\
= & \left|T_{1}\right|+\max \{0, w(3,2)-(w(4,2) \cdot 1+w(1,2) \cdot 0)\} \cdot\left|T_{3}\right| \\
+ & \max \{0,1-w(1,2) \cdot 1-w(3,2) \cdot 1-w(4,2) \cdot 1\} \cdot\left|T_{4}\right| \\
= & 0.25+(0.8-0.5) \cdot 0.25+0 \\
= & 0.25+0.30 \cdot 0.25=\frac{13}{40}=U_{\sigma}(2)
\end{array}
$$

It follows that player 2 in $\sigma^{\prime}$ gains the same as $\sigma$. However, this does not imply that $\sigma$ is a Nash equilibrium, since there must be another alternation of the player (or some other player) which results in a higher utility.

Similarly, the reader may attempt to evaluate alternate profiles of the game, by switching the operations mode of one node and re-evaluating the utilities of the players in a similar fashion as demonstrated above. We do not attempt to show the reader how to find the Nash Equilibrium for the particular topology at this time. However, such generalization remains within our future work goals.

\section{Conclusion}

The chapter has presented the use of graphical game theory towards the investigation and resolution of the following communication networking problem: Interference may arise using interactions between wireless access points that operate in the same geographical region without any coordination. Using a graphical game theoretic model, it has been shown that the players are motivated to create alliances with their neighbours so as to serve their terminals jointly and in a coordinated manner, leading to the decrease or even elimination of interference for all cooperating neighbours, an important achievement given the potential gains in Quality of Experience that the proposed framework can provide. The theoretical analysis shows the value of the cooperation for the interacting neighbours to form such a cooperative neighbourhood.

The cooperative neighbourhood model is represented as a game on a graph where the outgoing links of each node represent the signal strength (or interference depending on whether the node receiving the signal is $O N$ or $O F F$ ) received from a neighbour, and consequently quantifies the satisfaction that may result from cooperation. The theoretical analysis and the equilibrium between two players interacting in a cooperative neighbourhood, is just the first step towards the resolution of the game in a more generalized way. This first 
step towards the more generalized cooperative neighbourhood solution, has shown us that graphical games are a promising approach of modelling such interference situations.

In addition to the generalization of the theoretical work, the authors recognize that there exist more practical protocol design issues such as synchronization and dynamic node update issues, and are to be looked at as a part of future work issues. Furthermore, the practical application of the weight setting in a real world scenario also remains in the scope of future work. On the theoretical side, we plan to specifically seek equilibriums in more complex graphical representations of cooperative neighbourhoods represented as multi-player games. The considered example profile of a cooperative neighbourhood game, which simplifies the proposed cooperation in a four-node topology, illustrates the management of the $O N$ and OFF times of each server (AP) and how a profile can be modified. However, future work plans to further investigate the generation of equilibrium profiles in larger and more complex topologies, e.g. with partial neighbourhood relationships among the APs (i.e. where ranges of the individual APs overlap only partially), giving more general cooperative solutions.

\section{Author details}

Antoniou Josephina

School of Computing \& Mathematics, UCLan (University of Central Lancashire) Cyprus, Pyla, Cyprus

Lesta Papadopoulou Vicky

Department of Computer Science and Engineering, European University Cyprus, Nicosia, Cyprus

Libman Lavy

School of Computer Science and Engineering, University of New South Wales, Sydney, Australia

Pitsillides Andreas

Department of Computer Science, University of Cyprus, Nicosia, Cyprus

\section{References}

[1] J. Antoniou, and A. Pitsillides, Game Theory in Communication Networks: Cooperative Resolution of Interactive Networking Scenarios, ISBN: 987-1-4398-4808-1, CRC Press, Taylor \& Francis Group, Boca Raton, 2013.

[2] N. Nisan, T. Roughgarden, E. Tardos and V. V. Vazirani, Algorithmic Game Theory, ISBN: 978-0-521-87282-9, Cambridge University Press, New York, USA, 2007.

[3] A. Akella, G. Jedd, S. Seshan and P. Steenkiste, Self-Management in Chaotic Wireless Deployments, In ACM MobiCom, pp. 185-199, 2005.

[4] A. Hills, Large-Scale Wireless LAN Design, IEEE Communications vol. 39, no. 11, pp. 98-104, November 2001.

[5] Intel Research Seattle, Place Lab A Privacy-Observant Location System, http:/ / placelab.org/, 2004.

[6] O. A. Dragoi and J. P. Black, Enabling Chaotic Ubiquitous Computing, Technical Report CS-2004-35, University of Waterloo, Canada, 2004.

[7] P. A. Frangoudis, D. I. Zografos and G. C. Polyzos, Secure Interference Reporting for Dense Wi-Fi Deployments, Fifth International Student Workshop on Emerging Networking Experiments and Technologies, pp. 37-38, 2009. 
[8] J. Hassan, H. Sirisena and B. Landfeldt, Trust-Based Fast Authentication for Multiowner Wireless Networks, IEEE Transactions on Mobile Computing, vol. 7, no. 2, pp. 247-261, 2008.

[9] A. van de Nouweland, P. Borm, W. van Golstein Brouwers, R. Groot Briunderink and S. Tijs, A Game Theoretic Approach to Problems in Telecommunication, Management Science, vol. 42, no. 2, pp. 294-303, February 1996.

[10] A. Orda, R. Rom and N. Shimkin, Competitive Routing in Multiuser Communication Networks, IEEE/ACM Transactions on Networking, vol. 1, no. 5, pp. 510-521, 1993.

[11] A. de Palma, A Game Theoretic Approach to the Analysis of Simple Congested Networks, The American Economic Review, vol. 82, no. 2, pp. 185-199, 2005.

[12] L. Lopez, A. Fernandez and V. Cholvi, A Game Theoretic Comparison of TCP and Digital Fountain based protocols, Computer Networks, vol. 51, pp. 3413-3426, 2007.

[13] S. Rakshit and R. K. Guha, Fair Bandwidth Sharing in Distributed Systems: A Game Theoretic Approach, IEEE Transactions on Computers, vol. 54, no. 11, pp. 1384-1393, November 2005.

[14] H. Yaiche, R. R. Mazumdar and C. Rosenberg, A game theoretic framework for bandwidth allocation and pricing in broadband networks, IEEE/ACM Transactions on Networking, vol. 8, no. 5, pp. 667-678, 2000.

[15] J. Antoniou, I. Koukoutsidis, E. Jaho, A. Pitsillides, and I. Stavrakakis, Access Network Synthesis in Next Generation Networks, Elsevier Computer Networks Journal Elsevier Computer Networks Journal, vol. 53, no. 15, pp. 2716-2726, October 2009.

[16] J. Antoniou, V. Papadopoulou, V. Vassiliou, and A. Pitsillides, Cooperative User-Network Interactions in next generation communication networks, Computer Networks, vol. 54, no. 13, pp. 2239-2255, September 2010.

[17] J. Antoniou, L. Libman, and A. Pitsillides, A Game-Theory Based Approach To Reducing Interference In Dense Deployments of Home Wireless Networks. In proceedings of 16th IEEE Symposium on Computers and Communications (ISCC 2011), June 2011.

[18] G. Kendall, X. Yao and S. Y. Chong, The Iterated Prisoner's Dilemma: 20 Years On, Advances In Natural Computation Book Series, vol. 4, World Scientific Publishing Co., 2009.

[19] R. M. Axelrod, The Evolution of Cooperation, BASIC Books, New York, USA, 1984.

[20] M. Nowak, A. Sasaki, C. Taylor and D. Fudenberg, Emergence of cooperation and evolutionary stability in finite populations, Letters to Nature, vol. 428, April 2004.

[21] W. M. Grossman, New Tack Wins Prisoner's Dilemma, http://www.wired.com/culture/lifestyle/news/2004/10/65317, October 2004.

[22] A. Rogers, R. K. Dash, S. D. Ramchurn, P. Vytelingum and N. R. Jennings, Coordinating team players within a noisy Iterated Prisoner's Dilemma tournament, Elsevier Theoretical Computer Science, vol. 377, pp. 243-259, 2007.

[23] G. Taylor, Iterated Prisoner's Dilemma in MATLAB , Archive for the "Game Theory" Category "http://maths.straylight.co.uk/archives/category/game-theory", March 2007. 